\title{
Le pouvoir démiurgique d'un critique : Arthur Buies, personnage de Claude-Henri Grignon ${ }^{1}$
}

\author{
Jonathan Livernois \\ Université d'Ottawa
}

À Jimmy Labelle

Le purgatoire fut long et pénible pour celui que le sociologue Jean-Charles Falardeau considérait, en 1960, comme «notre Camille Desmoulins » (1960, p. 25). Il a fallu attendre les années 1970 et 1980, ponctuées notamment par les travaux de Georges-André Vachon, de Sylvain Simard, de Jean-Pierre

1 Une première version de ce texte a été présentée lors du Colloque « La critique littéraire et ses œuvres », organisé par Karim Larose et Karine Cellard au CRILQ-Université de Montréal le 5 mars 2010. 
Tusseau et de Francis Parmentier, pour que la critique prenne enfin la mesure pleine et entière de celui qui, selon Laurent Mailhot, « est le plus souple, le plus engagé, le plus complet des écrivains du XIXe siècle ici » (1994, p. 9). Cela n'empêcha pas Robert Vigneault d'écrire, en 1972 :

La pensée n'était pas née au Québec à cette époque; il n'y a eu alors que des simulacres de pensée ou des témoignages de notre aliénation intellectuelle, de caricatures de pensée qui nous font aujourd'hui sourire ou parfois même rire aux éclats. Il y a bien les vociférations de Buies, sorte de haut-le-cœur finalement pitoyable. (1972, p. 64)

Comment expliquer une telle fortune littéraire? On pourrait d'abord invoquer la difficulté, depuis au moins Étienne Parent ${ }^{2}$, de considérer la frivolité comme une valeur cardinale de la littérature du cru. Voilà qui faisait dire à François Ricard, relisant les chroniques d'Edmond Paré, que les Québécois ont «toujours quelque peine à considérer comme pleinement significatives les œuvres qui transgressent cette règle de l'utilité ou de la représentativité » (1991, p. 89). Nul doute que l'œuvre de celui qui s'est lui-même décrit comme « un oisif, un propre à rien, un déclassé » (Buies, 1986, p. 211) a souffert de ce soi-disant caractère atavique, comme l'a d'ailleurs rappelé Sylvain Simard ${ }^{3}$. À cela, il faut ajouter une autre explication, qui

\footnotetext{
2 «J'ai un autre regret, c'est que nos autres journaux canadiens ne reproduisent pas ces articles, ou ne dévouent pas tous, depuis quelque temps, une partie de leur espace à des analyses ou extraits de bons ouvrages sur l'économie politique. Une pareille matière, à mon humble avis, vaudrait bien les romans et nouvelles, plus ou moins frivoles, qu'ils nous débitent à la brasse dans chacune de leurs feuilles. Il faut à une population comme la nôtre, située comme la nôtre l'est, des lectures utiles et instructives. » (Parent, 2000, p. 121)

3 «Ceux qui ont voulu occulter la critique de Buies ont toujours insisté sur l'aspect souvent léger et pétillant de son œuvre afin d'en diminuer l'impact. » (Simard, 1981, p. 265)
} 
relève de l'histoire culturelle québécoise : l'intégration d'Arthur Buies au téléroman Les Belles Histoires des Pays d'en Haut, écrit par Claude-Henri Grignon. Si le purgatoire du pamphlétaire a été long, on peut certainement croire que la confusion entre la fiction et le réel, qui a marqué la mémoire culturelle québécoise, n'y a pas été étrangère. Buies est pour ainsi dire disparu sous la fiction et ses traits se sont effacés au profit de ceux du comédien Paul Dupuis, qui l'interpréta au petit écran de 1956 à 1962.

Plusieurs notèrent cet effacement de l'homme ou, du moins, le fait qu'il fut «doublé » par un alter ego fictif créé, de surcroît, par ce qu'il convient de considérer comme un ennemi idéologique : Claude-Henri Grignon. Ainsi, en 1978, lors de la publication d'une sélection de textes de Buies préparée par Laurent Mailhot, Robert Melançon écrivait dans Le Devoir : «Pour la plupart le nom d'Arthur Buies évoque d'abord un personnage de l'interminable, de l'insipide radio-télé-roman de Claude-Henri Grignon, Les Belles Histoires des Pays d'en Haut. C'est bien dommage.» (1978, p. 14) Quelques années auparavant, Jean Éthier-Blais écrivait :

Arthur Buies connut le sort des pamphlétaires fatigués et affamés; il se rangea. Le curé Labelle se trouva opportunément sur le chemin de Damas. Il releva le cavalier qui enfourcha la monture de la colonisation. Ironie du destin! Buies le réformateur consacra sa vie d'homme mûr à soutenir l'une des causes les plus néfastes de notre histoire : celle du peuplement du Nord. Il est devenu un personnage de la télévision, prêchant le retour à la terre... (1967, p. 107)

En 1986, Francis Parmentier, responsable de la réédition des Chroniques dans la "Bibliothèque du Nouveau Monde", ajoutait : «celui qui devait l'immortaliser à la télévision sous les traits du secrétaire du curé Labelle fut aussi l'un de ses plus 
impitoyables détracteurs » (dans Buies, 1986, p. 41). En effet, pour Claude-Henri Grignon, il ne semble pas y avoir suffisamment de procédés rhétoriques pour jeter l'anathème sur le chroniqueur. Cela ne surprend guère: Valdombre s'est attaqué à un grand nombre d'écrivains dans ses pamphlets, y publiant des « jugements qui louangent ou vitupèrent avec une semblable intempérance du langage» (Hamel, Hare et Wyczynski, 1989). Si Olivar Asselin et Alfred DesRochers semblent du bon côté, il n'en va pas de même pour Victor Barbeau ou pour Arthur Buies, par exemple. Dans ce dernier cas, Grignon va très loin : en 1963, il dit avoir tué Buies pour mieux le « ressusciter» dans sa propre œuvre de fiction. Voilà qui révèle le pouvoir potentiellement démiurgique d'un critique doublé d'un écrivain.

Dans cette étude, nous nous attacherons à trois textes qui façonnèrent l'image de Buies selon Grignon. Nous nous intéresserons ensuite au télescopage du réel et de la fiction dans le téléroman Les Belles Histoires des Pays d'en Haut, du moins dans les quelques épisodes encore existants où apparaît le personnage de Buies, entre 1956 et $1962^{4}$. L'objectif : mieux cerner l'image d'un Buies en décalage avec son identité réelle; comprendre l'effet de ce travail fictionnel sur la mémoire culturelle québécoise.

\footnotetext{
4 Depuis 2008, Bibliothèque et Archives nationales du Québec possède les textes des émissions de 1956 à 1970 (MSS246). Si leur analyse peut être, dans le cadre d'un travail éventuel, tout à fait pertinente, nous avons préféré nous attacher ici aux enregistrements encore existants, assez nombreux pour fixer un portrait du personnage. Nous considérons que l'image de Buies tient autant à l'interprétation - et à la vie - de Paul Dupuis qu'aux textes de Grignon.
} 


\section{Trois textes sur Buies}

Francis Parmentier, dans son édition critique des Chroniques, relève trois titres de Grignon qui s'attaquent au pamphlétaire : "Arthur Buies, ce fils Homais", paru dans la Minerve du 17 juillet 1920; ensuite, un extrait d'Ombres et clameurs, ces « regards sur la littérature canadienne» parus en 1933 chez Albert Lévesque; finalement, "Arthur Buies ou l'homme qui cherchait son malheur ", publié dans les Cahiers de l'Académie canadienne-française en 1963. Malgré les quarante-trois années qui séparent le premier et le dernier de ces textes, il y a une volonté forte qui ne change pas chez le critique : fixer à jamais l'image d'Arthur Buies. Grignon ne semble pouvoir souffrir le statut incertain de Buies, écrivain tantôt ironique, tantôt sérieux, tantôt chroniqueur impétueux, tantôt apôtre de la colonisation. Dans son texte de juillet 1920, Grignon traduit cette incertitude en projetant l'essayiste dans une sorte de purgatoire. Buies n'est pas tout à fait mort, mais n'est pas tout à fait vivant non plus. Une série d'images, presque gothiques, donne le ton : «Une tête drôle [...] pour illuminer la mort dans la nuit littéraire canadienne "; "l'épouvante vient de la Lanterne »; "L'épouvantail qui n'épouvante plus personne »; "Cette vieille tête que l'on craint d'enterrer »; "Les baves [de Buies] sont complètes et extrêmement écœurantes »; "des malades désespérés [...] cherchent avec des lanternes la rose agonisante », c'est-à-dire la chronique, dans un « tas de fumier » (Grignon, 1920, p. 2), c'est-à-dire dans La lanterne. Dans ce purgatoire, un peu comme si le demi-mort était désespéré de ne pas être tout à fait mort et comme s'il voulait en finir, il y a même de l'autophagie, fût-elle symbolique: «[Buies] a consommé son nom. Songez à cela. La libre-pensée n'a eu qu'à 
jeter un $e$ (qui n'est pas muet) dans le mot buis. » (Grignon, 1920, p. 2) Grignon joue ici sur les mots et sur l'homonymie buis/buies. Il marque le passage du buis, branche d'arbuste bénite le jour des Rameaux, telle une synecdoque des traditions anciennes de la civilisation chrétienne, à ce « buies » dont le « $e$ n'est pas muet », c'est-à-dire à ces cruches ou ces vases qui servaient autrefois, en France, à mettre de l'eau. Bref, la pensée anticléricale de l'essayiste l'a dévoré et n'a laissé que du vide, qu'une présence en creux. Et, si l'on en croit Grignon, cette pensée est largement répandue, ce qui conduit ce dernier à écrire ceci, tout à fait typique de cette « vision crépusculaire du monde » (Cellard, 2009, p. 241) que Karine Cellard associe à l'écriture des Pamphlets de Valdombre:

La fête du buis! Fête religieuse, (sans tambours et sans maire), fête surtout religieuse, très simple, capable de sauver toutes les âmes, celles d'aujourd'hui qui sombrent silencieusement dans la mer la plus agitée, la plus effrayante qu'il ait été accordé aux hommes de voir.

Non seulement en France. Non seulement chez les vieux peuples usés, fatigués, meurtris. Partout maintenant, partout, partout. La bénédiction du buis qui symbolisait autrefois le pardon éternel, doit sombrer aussi, devant les peuples effrayés. (Grignon, 1920, p. 2)

Ainsi faut-il en finir avant qu'il soit trop tard. Par un grand geste de violence, le demi-mort doit être enterré de manière définitive, comme sa pensée anticléricale : il faut sortir la lanterne « de l'oubli, d'un coup de plume ou d'épée. Que l'on permette, une fois, au bon sens, au vrai sens critique de renfoncer la misérable enseigne de lanterne dans le fumier de l'oubli comminatoire. Cette fois-là Buies ne sera plus. » (Grignon, 1920, p. 2) 
En 1963, on retrouve la même violence chez le critique : Grignon rappelle avoir, dans sa prime jeunesse, «saign[é] d'une main impitoyable » Arthur Buies et évoque sa propre " cruauté de sicaire» $(1963$, p. 30). L'exécution est encore une fois sommaire, tout aussi rapide que cette phrase simple, en retrait dans le texte: "Je tuai Buies ». Contrairement au texte de la Minerve, mais aussi à celui d'Ombres et clameurs qui reprend l'essentiel du précédent (une même association dangereuse avec Voltaire, le «vieil avare sensuel de Ferney», et avec Homais, le personnage de Flaubert), cet assassinat est suivi d'une résurrection. Le critique Grignon a désormais un pouvoir démiurgique étonnant. Il le répète par deux fois: "Je l'ai ressuscité! Voulez, voulez pas, c'est ça. Je l'ai ressuscité! » (1963, p. 31) Il dit avoir amené « Arthur Buies par la main non seulement au portique des Laurentides mais tout drette dans les Belles histoires des pays d'en haut à la télévision ». Résultat : l'écrivain du 19e siècle « vivra aussi longtemps dans la mémoire de [s]es compatriotes que le curé Labelle ou Séraphin, ce qui n'est pas peu dire » (1963, p. 31). La lanterne n'y fera rien : c'est l'image du comédien Paul Dupuis qui s'imposera. Mais, justement, quelle est l'image que donne Les Belles Histoires des Pays d'en Haut d'Arthur Buies? Est-elle carrément négative? La question se pose d'autant plus qu'on remarque dans le texte des Cahiers de l'Académie canadienne-française, à peu près contemporain de la fictionnalisation d'Arthur Buies, une sorte d'apaisement critique. Tout se passe comme si le pamphlétaire, désormais établi et n'ayant plus à se faire un nom, ne craignait plus la «concurrence» de ses prédécesseurs. La clémence pourrait même être un signe de supériorité ou de suffisance. Ainsi, rappelant d'abord son impétuosité de l'époque de la Minerve, réduisant ensuite Buies au romantisme et à 
l'anticléricalisme, Grignon est finalement plus clément avec Buies, même si « cet écrivain de gauche-là [le] désespère et [le] dégoûte» (1963, p.36). Il va même jusqu'à renverser son appréciation du style de Buies, considéré en 1933 comme "romantique, surchargé d'épithètes, de métaphores incohérentes, de verbes boiteux, de fautes de langue et toujours cette touchante imprécision» (1933, p.90). Trente ans plus tard, sa « langue » est devenue « sûre et directe » (1963, p. 41). C'est aussi avec émotion que Grignon relate la rencontre entre le curé Labelle et le pauvre homme solitaire, ce qui annonce la rédemption du pécheur ou son chemin de Damas. À en croire Valdombre, les deux hommes ne sont rien de moins que les deux grands piliers de la colonisation du Nord. Au contact du bon curé, l'anticlérical a même redécouvert Dieu: «je veux croire, j'en ai même la certitude, que l'anticlérical Buies se réconcilia avec Dieu avant son dernier soupir dans la nuit froide du 21 janvier 1901. Il trouvait enfin le Bonheur, la Joie éternelle et suprême! » (1963, p.41) C'est cet homme, l'ami du curé Labelle qui sera bientôt rédimé par l'œuvre de la colonisation et par l'attachement à la terre, que découvrent les téléspectateurs des Belles Histoires des Pays d'en Haut, dont les épisodes seront diffusés une première fois entre 1956 et 1970 et à de multiples reprises par la suite. À peindre un homme foudroyé sur son cheval, on oublie, volontairement ou non, qu'il a longtemps chevauché.

Dans Les Belles Histoires des Pays d'en Haut, le personnage d'Arthur Buies, secrétaire du curé Labelle, est interprété par Paul Dupuis (1916-1976), acteur bouillant dont le destin n'est pas sans rappeler celui de son personnage - nous y reviendrons. Présent dès les débuts de la série, Dupuis la quittera en 1962 dans des circonstances nébuleuses : certains 
attribuent ce départ à un conflit avec Grignon lui-même (voir Bertrand, 2003, p. 198-199). Il sera remplacé par le comédien Raymond Royer, qui refusera de reprendre le rôle de Buies et proposera plutôt de créer un personnage fictif, le secrétaire Dubouquet. Quoi qu'il en soit, durant six années, Buies marque l'imaginaire québécois.

\section{Les femmes et la frivolité plutôt que La lanterne}

Dès le premier épisode de la série ("L'assemblée politique »), diffusé le 8 octobre 1956 et présenté par Claude-Henri Grignon comme une "peinture réaliste d'un passé qui ne peut pas mourir ${ }^{5}$ », le décor est planté : le curé Labelle n'existe pas sans Arthur Buies et Arthur Buies n'existe pas sans le curé Labelle. En ce mois d'octobre 1886, Honoré Mercier, interprété par le comédien Pierre Boucher, fait un discours devant la population de Saint-Jérôme. La fenêtre du presbytère est ouverte et les deux hommes se disputent à propos du discours. Si Buies est déjà conquis par Mercier (il dit : «Écoutez donc, curé : Mercier sait ce qu'il dit! »), la promesse de donner une terre aux pères de douze enfants vivants et plus vient changer d'un coup l'humeur du curé Labelle, plutôt bleu (politiquement), qui invite Mercier à souper. Le dialogue qui s'ensuit entre Mercier, Buies et deux personnages fictifs, le docteur Cyprien et la belle Angélique, permet déjà de limiter, subrepticement, l'influence du pamphlétaire :

5 Toutes les références aux épisodes sont tirées d'une série de 20 DVD («Les Belles Histoires des Pays d'en Haut») présentée par la Société RadioCanada et par Imavision en 2008. 
Mercier : Ah, mais tiens! Si ce n'est pas ce cher Buies qui vient parici.

Angélique : Buies? Vous parlez d'Arthur?

Mercier : Mais oui! Le fameux pamphlétaire!

Docteur Cyprien : Le dangereux...

Mercier: Oh! Il se radoucira bien.

Buies : J'ai le plaisir de vous féliciter, monsieur le premier ministre.

Mercier : Pas trop vite, pas trop vite. Attendez au moins après le 14.

Buies : Bon, je vais vous dire tout simplement que vous nous avez définitivement gagnés à votre cause, le curé Labelle et moi. Mercier: Un instant, mon cher. Permettez-moi de vous présenter l'amie de mon ami, le docteur Cyprien, madamoiselle Angélique Pothier. Monsieur Arthur Buies, journaliste.

Buies : Mes hommages, mademoiselle.

Angélique : Et toutes mes félicitations à l'écrivain.

Buies : Ah! Vous voulez-dire au pamphlétaire!

Angélique : Non, non, non, pas à l'auteur de La lanterne. Je m'adresse au chroniqueur, au signataire des Caprices.

Buies : Vous avez lu ça?

Cyprien : Mais vous savez bien que toutes les femmes vous lisent. Et quand on est lu par les femmes, c'est déjà la postérité!

Buies : Postérité, peut-être, mais pas la richesse!

Dès le départ, le destin du pamphlétaire est pour ainsi dire scellé : le caractère "dangereux » de ses écrits antérieurs (résumés par La lanterne, dont la réédition réelle eut lieu en juin 1884) s'efface ou s'effacera bientôt («Oh! Il se radoucira bien », dit Mercier) au profit d'une littérature dite mineure, légère, sans conséquence apparente (résumée par ses Chroniques). Ainsi l'assertion du docteur Cyprien («Vous savez bien que toutes les femmes vous lisent ») a-t-elle pour effet de reléguer l'écrivain dans le domaine de la frivolité, du divertissement léger pour dames de la bonne société. On sait la fortune, au Québec, de la littérature légère, frivole. 
Les propos du docteur Cyprien sur le lectorat féminin de Buies font en quelque sorte écho à ceux que Grignon tenait en 1920 dans La Minerve : "L'allumeur de la Lanterne a écrit sans fatigue des pages sans intelligence, mais dans lesquelles on pourrait encore trouver de l'esprit, de l'esprit prime-sautier, comme disent les bas-bleus entre eux, quand les bas-bleus sont seul(es), entre elles. » (1920, p. 2) L'intertexte flaubertien étant annoncé dès le titre de l'article, on retournera volontiers au Dictionnaire des idées reçues : «Bas-bleu. — Terme de mépris pour désigner toute femme qui s'intéresse aux choses intellectuelles. » (Flaubert, 1966, p. 337) Voilà qui ne sied pas mal à la personnalité de la belle Angélique, bourgeoise perdue en territoire de colonisation. Sera-t-on surpris d'apprendre, dans l'épisode du 16 octobre 1961 ( «a maison de l'exilé »), que cette bonne dame a entretenu pendant cinq ans une relation épistolaire avec Buies? Comme le dit le curé Labelle à son secrétaire : « On se fait toujours prendre, hein, Arthur? »

Présente dès le départ, donc, comme un gage de frivolité et de badinage, la gente féminine sera aussi responsable de l'assagissement final de l'écrivain impétueux. Dans l'épisode du 17 septembre 1962, tandis que le comédien Paul Dupuis a déjà quitté la série, on apprend que Buies n'est plus au service du curé Labelle et a épousé une demoiselle Marie-Mila Catellier6. C'est par le truchement du personnage du curé Labelle que Grignon vient ainsi fixer le portrait de l'essayiste ou, pour reprendre le vocabulaire du jeune Valdombre, enterrer le crâne de l'anticlérical. Le curé, malgré le « dur coup» que signifie le

${ }^{6}$ Ce fait est tout à fait réel : comme le rappelle Francis Parmentier (Buies, 1986, p. 63), Arthur Buies a épousé Marie-Mila Catellier, fille de Ludger-Aimé Catellier, « registrateur général adjoint du Canada à Ottawa, le 8 août 1887. 
départ d'un "secrétaire en or», déclare en effet: "Mais aujourd'hui, Buies est un homme rangé. Je suis certain qu'il sera un mari modèle. » La vie conjugale vient d'achever l'homme de lettres. Mais, avant de tourner la page, le curé Labelle repousse de nouveau La lanterne: "Les historiens, les fins-fins de la critique pourront lui reprocher sa Lanterne, cruelle, et parfois son style, grandiloquent. Mais moi, notaire [Le Potiron], moi qui l'ai vu à l'œuvre, je dois vous dire que Buies apportait dans son métier une conscience professionnelle qui l'honore. »

\section{Arthur Buies, le converti?}

On pourrait s'étonner du fait que Grignon ne peint pas Arthur Buies, dans Les Belles Histoires des Pays d'en Haut, comme un converti, d'autant plus que celui-ci, dès 1879, semble redécouvrir Dieu. À l'époque, Buies écrivait à Alfred Garneau : «Depuis le jour où j'ai communié de nouveau après 23 ans d'abstention, je n'ai pas éprouvé pareil soulagement. » (1986, p. 58) Pourtant, dans le téléroman de Grignon, Buies fait encore figure d'anticlérical, quoique particulièrement adouci. Dès le premier épisode de la série, le curé Labelle l’interpelle : «Toi, libre-penseur, toi aimer Jésus? Hypocrite! Tu n'es qu'un anticlérical! » Comment expliquer de telles répliques? Tout se passe en fait comme si Grignon, jouant de nouveau sur les faits historiques, maintenait le pôle d'opposition anticlérical pour mieux le désamorcer. L'anticléricalisme de Buies, longtemps d'une rare violence - Michel Biron rappelle que l'homme «bouffait du curé comme cela n'était permis de le faire dans le Canada français de Mgr Bourget» (2010, p. 39) —, n'est plus qu'une source de désaccords anodins, voire de taquineries, 
entre le secrétaire et celui qu'il appelle tout simplement "curé ». Par exemple, au début de l'épisode du 16 octobre 1961, l'ecclésiastique se moque de Buies, lui parle de son "auréole future» et de "saint Arthur Buies»; il va même jusqu'à lui frotter la tête comme on le ferait à un enfant pour le taquiner.

Cela dit, Grignon n'est peut-être pas très loin de la réalité historique lorsqu'il présente son anticlérical dans le presbytère de Saint-Jérôme : comme l'a bien montré Jean-Pierre Tusseau, "[l]es "rechutes" sont trop fréquentes et surtout trop cohérentes, trop orientées pour qu'il ne soit pas frauduleux de parler de conversion vers 1880 »; « [l]'épisode du curé Labelle, que [Tusseau ne remet] pas en cause, n'est pas un dénouement mais un intermède» (Tusseau, 1973, p. 52). Grignon, subrepticement, ne refuse pas ici d'envisager la conversion, qu'il évoque dans son texte de 1963: il désamorce l'anticléricalisme pour mieux dégager le chemin de Damas, jamais montré, mais que le téléspectateur devine, dès le début de la série. Ainsi, dans le premier épisode, avant que le secrétaire disparaisse pour écrire toute la nuit « la vérité, toute la vérité », le curé Labelle lui remet un chapelet. Buies joue avec l'objet, la caméra fait un gros plan sur celui-ci tandis qu'on entend la voix de l'anticlérical : «Un chapelet... Sait-on jamais?... Un chapelet... » Au téléspectateur de deviner jusqu'où ira l'homme.

\section{Contamination du réel par la fiction... et par le réel}

À tout prendre, le travail de Grignon, dans son téléroman, n'est plus celui du pamphlétaire : c'est celui, plus retors peut-être, de 
l'écrivain qui désamorce la charge explosive d'une œuvre qu'il abhorre. D’abord, il a désamorcé La lanterne, effacée par les Chroniques - que Grignon, dans son texte de 1920, comparait à une "rose agonisante» - et par des divertissements légers pour « bas bleus». Il a aussi cantonné l'essayiste dans la seconde partie de sa vie tout en prenant soin de "ramener» son anticléricalisme. Ce dernier, devenu puéril, est réduit à des querelles aussi subites que fugaces entre le curé Labelle et son secrétaire. À cela, on pourrait ajouter l'impression que Grignon désamorce l'œuvre en plongeant l'homme dans des intrigues mineures qui finissent par occuper toute la place, en le situant dans une galerie de personnages qui ont une influence certaine sur son image. On pourrait parler d'une «contamination» du réel par la fiction.

On notera d'abord une association indirecte du secrétaire du curé Labelle avec le personnage fictif d'Alexis Labranche, l'exilé et ennemi juré de Séraphin Poudrier. Les problèmes d'alcool de l'exilé au grand cœur, draveur courageux et bel homme, soulignent ceux de Buies, bien réels ${ }^{7}$, que l'on ne devine que par quelques propos du curé Labelle - ainsi, dans l'épisode du 1er juin 1959, "Rencontre orageuse entre Alexis et le grand William », l'ecclésiastique dit à son secrétaire : "Tu peux en parler, toi, Arthur, des ivrognes convertis! On connaît ça!» L'association entre Alexis et Buies permet aussi de mettre l'accent sur le caractère aventureux du second, qui ressort clairement dans le téléroman. Dans l'épisode du 5 octobre 1959

\footnotetext{
${ }^{7}$ Comme le rappelle Parmentier (Buies, 1986, p. 60), le curé Labelle écrivait à Buies le 6 décembre 1882: "J'ai appris à Montréal, avec regret, que vous aviez oublié vos bonnes résolutions. On me rapporte que parfois mon vicaire s'oublie un peu trop. Je dois vous dire qu'il y a déjà des plaintes contre vous au sujet de l'intempérance. »
} 
(«Alexis joue à cache-cache »), le secrétaire du curé Labelle va jusque dans un saloon du Colarado pour retrouver le pauvre Alexis, qui s'est enfui de Saint-Adèle à la suite d'un accident mortel. Il va sans dire qu'il faut aimer un homme pour traverser l'Amérique depuis plusieurs mois afin de le retrouver et de le rapatrier...

Toujours enclin à quelque cavalcade à travers les Laurentides ou à travers le far-west du Colorado, Buies, tout comme Alexis, porte le chapeau de l'aventurier. Le costume a son importance: même dans le presbytère de Saint-Jérôme, Buies délaisse la cravate pour porter un habit qui rappelle autant un explorateur européen en Afrique qu'une version fin 19 e du coureur des bois. C'est sur ce trait que passe et repasse Grignon: Buies est un aventurier qui délaisse les grands pamphlets pour vivre à plein les événements, même les plus insignifiants, de la colonisation du Nord. Dans l'épisode du 26 mars 1962, le pamphlétaire gage son " dernier 10 piastres » dans une course de chevaux sur glace à laquelle il participera, ce qui fait dire au curé Labelle : "T'as toujours aimé le danger, le risque, prendre des chances... » Le risque, pour Buies, ce ne sont plus les grandes envolées contre le clergé ou la société canadienne-française : ce sont des paris anodins ou, dans cet autre épisode qui se déroule dans un saloon du Colorado (5 octobre 1959), la consommation d'un verre de rhum, liqueur qu'on dit «dangereuse». L'homme répond du tac au tac: «J'aime le danger ». La « ligne du risque » du pamphlétaire s'est pour ainsi dire déplacée de la grande scène sociale à la petite scène personnelle, prosaïque, mineure. L'aventurier est occupé par des querelles de clocher: par exemple, dans l'épisode du 6 novembre 1961 («La future maîtresse de poste»), il se passionne pour le choix de la future maîtresse de poste et 
envisage d'aller jusqu'à Ottawa pour voir [Joseph-Adolphe] Chapleau et pour défendre sa candidate. Le domaine de l'écrivain se serait-il réduit comme une peau de chagrin? C'est du moins l'impression que donne le portrait que s'évertue à tracer Claude-Henri Grignon.

Si la fiction contamine le réel, le réel peut aussi contaminer le réel. En effet, il semble bien que la vie du comédien Paul Dupuis vienne aussi doubler celle d'Arthur Buies, lui imposant cette image d'aventurier solitaire, voire asocial. Le lendemain de son décès, le 23 janvier 1976, le journal La Presse résumait ainsi la vie et la carrière du comédien: un des premiers Compagnons du Père Legault; correspondant de guerre en Angleterre pour Radio-Canada; carrière en Angleterre et en France; retour au Québec; animation qui fait scandale autant à Télé-Métropole qu'à CKVL, où les «autorités diocésaines" intervinrent contre lui; démission fracassante de l'Union des artistes; long voyage dans le Pacifique; décès à 59 ans dans une chambre d'hôtel de SaintSauveur (Rudel-Tessier, 1976, p. 8). Cette vie, du moins pour le côté spectaculaire, ressemble à celle de Buies, qui a tout d'un «loose cannon» (Biron, 2010, p. 41): le pamphlétaire a beaucoup voyagé, a vécu en Europe, s'est attiré les foudres du clergé, s'est fait quelques ennemis en passant, est revenu au Nord pour écrire et pour mourir. Ce lien entre deux vies mouvementées a été effectué par Dupuis lui-même : en 1975, dans une émission de la série "Portraits» (radio de RadioCanada) consacrée à Arthur Buies, le comédien confiait avoir modelé sa vie sur celle de Buies. Effet de réverbération mémorielle : dès son enfance, l'écrivain aurait été pour lui son «Superman » (Portraits, 1975). Tandis qu'il passait ses étés à Sainte-Luce, il entendait l'amie de sa mère, Yvonne, la fille de 
Buies, ainsi que la veuve du pamphlétaire raconter leurs souvenirs de ce dernier. Ceux-ci l'ont marqué, à telle enseigne que le "Superman» de son enfance est devenu "son idéal d'homme », son «maître », dont il a lu " tout ce qui lui tombait sous la main » (Portraits, 1975). Lorsqu'on lui proposa le rôle à la télévision, il dit n'avoir eu qu'un but : faire aimer l'homme. En 1975, il croyait avoir réussi, tandis que des gens dans la rue le saluaient encore en lui demandant : «Comment ça va, Monsieur Buies? » Deux vies se sont bel et bien mêlées dans la mémoire culturelle québécoise.

\section{Buies : un autre homme après la Révolution tranquille?}

Claude-Henri Grignon a donc usé d'une sorte de pouvoir démiurgique: par le truchement de la fiction, il a tué un écrivain. Il l'a cantonné dans son rôle d'aventurier, d'homme qui serait passé de la critique véhémente à la passion pour les «belles histoires» qui ponctuèrent les saisons du Nord. Il a aussi tracé pour lui un chemin de Damas; il l'a relégué au rôle d'écrivain dont on devine certes l'activité polémique mais dont on voit surtout le côté badin et amoureux qui plaît tant aux «bas-bleus » comme la belle Angélique. Paul Dupuis, qui en aurait fait son maître, lui aura aussi imposé sa propre image d'homme asocial plus enclin à partir qu'à rester. Voilà qui explique pour une large part la place de Buies dans la mémoire culturelle québécoise.

L'auteur des Belles Histoires des Pays d'en Haut a trouvé une arme critique particulièrement efficace pour enterrer le crâne de l'anticlérical : la fiction. Fini, le purgatoire? Cela n'est peut-être pas aussi simple: Arthur Buies est aujourd'hui 
considéré, voire célébré par les historiens de la littérature et par «les fins-fins de la critique »; la courbe d'appréciation de son œuvre épouse en quelque sorte le mouvement de l'histoire intellectuelle québécoise, comme le donne à penser Michel Biron : «Quelques biographes se sont emparés avec délectation du personnage, d'abord pour condamner l'instable voltairien, puis, après la Révolution tranquille, pour célébrer au contraire ce libre-penseur. » (2010, p. 39) Encore une fois, c'est à la télévision que certains chercheront à marquer le changement. Il faut évoquer en ce sens le téléroman de Radio-Canada, Cormoran (1989-1993), qui raconte la vie d'une famille bourgeoise du Bas-Saint-Laurent. Cette série est l'œuvre de Pierre Gauvreau, signataire de Refus global situé à des kilomètres idéologiques de Claude-Henri Grignon. Dans l'avantdernier épisode, on a l'impression que Gauvreau cherche à réhabiliter Buies, à modifier l'image qui s'est imprégnée et qui persiste dans la mémoire culturelle québécoise, malgré l'effort critique des années 1970 et 1980. Voici la scène: Georges Legris, un avocat connu pour sa défense passionnée des marginaux, discute avec la famille Cormoran.

Georges Legris: Dans ces pays, le bonheur pèse sur les populations comme la lourde atmosphère des jours chauds qui endort toute la nature. Ce calme est plus effrayant que les échafauds où ruisselle le sang des Patriotes.

Docteur Pacifique Cormoran : Arthur Buies!

Georges Legris : Ah!Ah! Lui-même! Enfoui au fond d'un de nos fameux trous de mémoire. (Il trinque) À un avenir où tu retrouveras ta place, mon cher Arthur!

Ginette Cormoran : Bon, ben, les hommes avant que votre enthousiasme pour ce fameux Arthur que personne ne connaît vous fasse rouler sous la table ${ }^{8} \ldots$

8 Épisode rediffusé sur les ondes de ARTV le 24 novembre 2010. 
C'est par la fiction que la mémoire cherche à se frayer un chemin. Pourtant, les «trous de mémoire» persisteront et l'image imposée par Grignon demeurera. Comme le dit Ginette Cormoran, «personne ne connaît » le « fameux Arthur ».

Comment un personnage fictif pourrait-il ressusciter, dans la mémoire culturelle québécoise, tel un écrivain réel? Tâche ardue, d'autant plus que dans ce cas-ci, la culture québécoise semble préserver de vieilles habitudes canadiennes-françaises d'irréalisme, lesquelles ont donné une histoire providentielle - de Rameau de Saint-Père à Lionel Groulx - et des traits que dénonçait, il n'y a pas si longtemps, Pierre Vadeboncœur' ${ }^{9}$. Le cas d'Arthur Buies, esprit fascinant dont la mémoire culturelle ne garde pas grand-chose sinon le personnage de Grignon et quelques marques toponymiques, nous montre le choix d'une représentation fictive plutôt que celui d'une prise en compte du passé dont on a aujourd'hui les moyens d'obturer les «fameux trous de mémoire ». ClaudeHenri Grignon semble avoir eu raison: il a parié sur les habitudes d'un Canada français qui «ne peut pas mourir», du moins pas complètement.

\section{Bibliographie}

\footnotetext{
9 Il écrivait en 1951 : « Nous nourrissons des mystiques, des idéaux, dont la référence au réel ne se fait plus que vaguement : c'est là le trait le plus irritant de notre culture. Sur le plan politique, par exemple, la chose est évidente: nous entretenons un projet national, un thème hautement patriotique, sans qu'une réalité impérieuse ou exaltante fournisse les éléments essentiels d'une mystique politique dynamique possible. » (Vadeboncœur, 2007, p. 39)
} 
BERTRAND, Luc (2003). Un peuple et son avare. Sources et histoire d'un téléroman, Montréal, Libre Expression.

BIRON, Michel (2010). La Conscience du désert, Montréal, Éditions du Boréal.

BUIES, Arthur (1986). Chroniques I, édition critique par Francis Parmentier, Montréal, Presses de l'Université de Montréal, coll. « Bibliothèque du Nouveau Monde ».

CELLARD, Karine (2009). "Au terme de l'“âge de la critique": Valdombre et le chant du cygne du régionalisme ", dans Yvan Lamonde et Denis Saint-Jacques (dir.), 1937: un tournant culturel, Québec, Presses de l'Université Laval, coll. «Cultures québécoises », p. 239-254.

ÉTHIER-BLAIS, Jean (1967). «Enfer et ciel; aller-retour », dans Signets II, Ottawa, Le Cercle du Livre de France, p. 105-114.

FALARDEAU, Jean-Charles (1960). «Arthur Buies, l'antizouave », Cité libre, no 27, p. 25.

FLAUBERT, Gustave (1966). "Dictionnaire des idées reçues », dans Bouvard et Pécuchet, chronologie et préface par Jacques Suffel, Paris, GF, p. 333-378.

GRIGNON, Claude-Henri (1920). «Arthur Buies, ce fils Homais », La Minerve, 17 juil., p. 2.

- (1933). Ombres et clameurs. Regards sur la littérature canadienne, Montréal, Éditions Albert Lévesque.

- (1963). "Arthur Buies ou l'homme qui cherchait son malheur», Cahiers de l'Académie canadienne-française, no 7, p. 29-42.

HAMEL, Réginald, John HARE et Paul WYCZYNSKI (1989). Dictionnaire des auteurs de langue française en Amérique du Nord, Montréal, Fides, http://services.banq.qc.ca/sdx/dalfan/.

MAILHOT, Laurent, dans Arthur Buies (1994 [1978]). Anthologie, introduction et choix de textes par Laurent Mailhot, Montréal, Bibliothèque québécoise. 
MELANÇON, Robert (1978). "Avez-vous lu Arthur Buies?», Le Devoir, 29 juil., p. 14.

PARENT, Étienne (2000). Discours, édition critique par Claude Couture et Yvan Lamonde, Montréal, Presses de l'Université de Montréal, coll. « Bibliothèque du Nouveau Monde ».

Portraits (1975), entrevue radiophonique avec Paul Dupuis réalisée par Madeleine Gérome, diffusée le 29 avril, Société Radio-Canada, no 621950 du Centre d'archives GastonMiron, <http://www.crlq.umontreal.ca $>$

RICARD, François (1991). "Sur une idée de Léon Gérin ou de la littérature comme frivolité », Études françaises, vol. 27, no 3, p. 73-89.

RUDEL-TESSIER, Joseph (1976). "Paul Dupuis est mort», La Presse, 24 janv., p. B8.

SIMARD, Sylvain (1981). "L'essai québécois au XIXe siècle », Voix et images, vol. 6, no 2, p. 261-268.

TUSSEAU, Jean-Pierre (1973). "La fin "éditifiante" d'Arthur Buies », Études françaises, vol. 9, nº 1, p. 45-54.

VACHON, Georges-André (1970). "Arthur Buies, écrivain», Études françaises, vol. 6, no 3, p. 283-296.

VADEBONCCEUR, Pierre (2007). Une tradition d'emportement. Écrits (1945-1965), choix de textes et présentations par Yvan Lamonde et Jonathan Livernois, Québec, Presses de l'Université Laval, coll. « Cultures québécoises ».

VIGNEAULT, Robert (1972). «L'essai québécois : la naissance d'une pensée », Études littéraires, vol. 5, no 1, p. 59-73. 


\title{
Résumé
}

Écrivain majeur du 19e siècle canadien-français, Arthur Buies occupe une place étonnante dans la mémoire culturelle québécoise : ses écrits ont pour la plupart été oubliés et il est devenu un personnage du téléroman Les Belles Histoires des Pays d'en Haut; il est devenu le comédien Paul Dupuis, qui l'interpréta de 1956 à 1962 à la télévision. Cette étude met en relief le rôle de Claude-Henri Grignon dans cet « assassinat » et dans cette « résurrection » au cœur de la fiction.

\begin{abstract}
Important writer of the French-Canadian's 19th century, Arthur Buies has a unique place in Quebec's cultural memory: if his works were mostly forgotten, he became a TV's character during 6 years (1956-1962) in Les Belles Histoires des Pays d'en Haut; he became Paul Dupuis, the actor. We want to explain Claude-Henri Grignon's role in this " assassinat» and in this « résurrection » in the fiction
\end{abstract}

